\title{
Tumor-tailored treatment of colorectal cancer: from bench to bedside?
}

\author{
Marko Kornmann \\ From 16th International Charles Heidelberger Symposium on Cancer Research \\ Coimbra, Portugal. 26-28 September 2010
}

\section{Background}

A broad variety of systemic treatment options are available for non-resectable colorectal cancer in UICC stage IV disease. Depending on age, comorbidity, the possibility of a secondary resection, the personal experience with specific drug combinations, and national guidelines physicians choose a treatment option. At present, only Ki-ras status is determined as an individual biological marker for resistance to anti-EGF receptor treatment with cetuximab. The use of other molecular markers could so far not be transferred to routine clinical use as a guide for treatment of advanced colorectal cancer. Thymidylate synthase (TS) is a key enzyme in DNA synthesis and one major target of 5-fluorouracil (5-FU) treatment. Several studies demonstrated that tumors with high TS expression did not respond to 5-FU treatment, whereas tumors with high TS may still respond to irinotecan (CPT-11), a topoisomerase II inhibitor.

\section{Aim}

The aim of our study was to determine the value of pretherapeutic tumoral TS level for selecting chemotherapy.

\section{Methods}

We designed a phase II multicenter trial comparing first-line monotreatment with 5-FU/folinic acid (FA) and the combination of 5-FU/FA and irinotecan. A biopsy from a reference lesion of patients with stage IV non-resectable colorectal cancer planned to undergo systemic palliative chemotherapy was taken. Relative TS mRNA expression from microdissected tumor tissue was determined followed by stratification in TS low and TS high and subsequent randomisation to mono- or combination treatment. Primary endpoint was response to treatment.

\section{Results}

A total of 168 patients underwent TS determination. ITT analysis of response revealed that toxicity was higher in patients receiving combination treatment. Overall survival not influenced by TS or treatment. Response to the combination treatment was similar in the TS low and high group. In contrast, response to 5-FU monotherapy was higher in the TS low group in comparison to the TS high group.

\section{Conclusions}

Individual determination of intratumoral TS is save and feasible and can help to individualize systemic treatment of advanced colorectal cancer. 5-FU monotherapy may be an effective treatment option in patients with low TS levels.

\section{Acknowledgements \\ This study is presented on behalf of the Study Group Oncology for Gastrointestinal Tumors (FOGT).}

Published: 24 September 2010

\section{doi:}

Cite this article as: Kornmann: Tumor-tailored treatment of colorectal cancer: from bench to bedside? BMC Proceedings 2010 4(Suppl 2):O25. 